\title{
UTILIZAÇÃO DOS BENEFÍCIOS DAS DIMENSÕES DA GPTI E OS FATORES CRÍTICOS DE SUCESSO PARA ANALISAR IMPLEMENTAÇÕES DE SISTEMAS ERP ${ }^{1}$ \\ USING THE BENEFITS OF ITPM DIMENSIONS AND CRITICAL SUCCESS FACTORS FOR ANALYZING ERP SYSTEM IMPLEMENTATIONS
}

\author{
(artigo submetido em novembro de 2009)
}

Pietro Cunha Dolci

Depto. de Ciências Administrativas - Universidade Federal do Rio Grande do Sul (UFRGS)

pcdolci@gmail.com

\author{
Antônio Carlos Gastaud Maçada \\ Depto. de Ciências Administrativas - Universidade Federal do Rio Grande do Sul (UFRGS) \\ acgmacada@ea.ufrgs.br
}

\begin{abstract}
The analysis of ERP system implementation remains both, a source of great managerial interest and academic research. However, one of the challenges of these projects is to convince people of the benefits of having a unified management system, which requires efforts from various business units. Thus, the objective of this research is to understand what the IT and business managers' perception is about the various benefits and critical success factors that integrated systems can provide to their businesses. In order to identify the benefits, the dimensions of the IT portfolio management (ITPM) were used, likewise, to identify the critical factors, Somers and Nelson's model was used. To achieve the objective of this study, multiple cases were carried out in three organizations: a hospital complex, a network of pharmacies and a supermarket. In all examined cases, the companies had formal processes of choice and selection of potential suppliers. Regarding to the benefits that ERP systems provide, through the dimensions of ITPM, different benefits were identified within each dimension. It was identify that organizations 2 and 3 consider ERP systems more transactional, while organization 1 considers ERP systems more informational, regardless of the other dimensions. With respect to critical factors, there was also some difference in perceptions, but also some similarities: communication and interdepartmental cooperation, the support of top management, clear objectives/goals and the supplier support.
\end{abstract}

Key-words: benefits; ERP implementation; dimensions of ITPM; critical success factors.

\section{RESUMO}

A análise da implementação de sistemas ERP continua sendo fonte de grande interesse gerencial e de pesquisas acadêmicas. Entretanto, um dos desafios dos projetos relacionados a esses processos é convencer as pessoas dos benefícios de ter um sistema de gestão unificado, que exige esforços de diversas unidades de negócio. Dessa forma, o objetivo desta pesquisa é compreender como os gestores de TI e de negócio identificam os diferentes benefícios e fatores críticos de sucesso que os sistemas integrados podem proporcionar as suas empresas. Para identificar os benefícios foram utilizadas as dimensões da gestão de portfólio de TI (GPTI) e para os fatores críticos o modelo de Somers e Nelson. Foram realizados estudos de caso em três organizações: um complexo hospitalar, uma rede de farmácias e um supermercado. Em todos os casos analisados, as empresas possuíam processos formais de escolha e seleção dos potenciais fornecedores. Em relação aos benefícios que os sistemas ERP proporcionam utilizando as dimensões da GPTI, foram identificados diferentes benefícios dentro de cada uma das dimensões, podendo-se destacar que as empresas 2 e 3 consideram os sistemas ERP mais transacionais, enquanto a empresa 1 considera o sistema mais informacional. Com relação aos fatores críticos, as empresas apresentaram algumas diferenças, mas também há algumas similaridades, podendo-se destacar a comunicação e cooperação interdepartamental, o suporte da alta gerência, a clareza dos objetivos/metas e a necessidade de auxilio do fornecedor.

Palavras-chave: benefícios; implementação de ERP; dimensões da gestão de portfólio de Tl; fatores críticos de sucesso.

1 Os autores agradecem o apoio recebido da Capes e do CNPq. 


\section{INTRODUÇÃO}

Os benefícios de investir em sistemas de gestão empresarial, denominados ERP (do inglês, Enterprise Resource Planing), mesmo possuindo um importante desenvolvimento com a evolução e disseminação de tecnologias de informação (TI) nos anos 90, continua sendo fonte de grande interesse gerencial e de pesquisas acadêmicas (BERCHET e HABCHI, 2005). Em 2008, o mercado mundial de ERP movimentou cerca de US\$229,2 bilhões, um crescimento de 13,9\% em relação a 2007. Para 2009, a previsão era que esse mercado gerasse receitas de US\$244,3 bilhões, resultado 6,6\% superior a 2008 (TI INSIDE, 2008).

Apesar desses elevados valores, um dos desafios dos projetos relacionados à implementação de ERP é convencer as pessoas dos benefícios de ter um sistema de gestão unificado, que exige esforços de diversas unidades de negócio (COMPUTERWORLD, 2009). Existem diferentes pesquisas que avaliam os benefícios que as empresas obtêm, utilizando os sistemas ERP (SOUZA e ZWICKER, 2006; KOH, GUNASEKARAN E RAJKUMAR, 2008; MALHOTRA e TEMPONI, 2010; SU e YANG, 2010).

Então, pesquisas que avaliem os benefícios que os sistemas ERP proporcionam podem contribuir para diminuir os desafios enfrentados pelas empresas no momento de realizar a implementação desses sistemas. Morton e Hu (2008) apontam que os benefícios associados com os sistemas ERP são tangíveis e intangíveis, e podem refletir nas dimensões operacionais, gerenciais, estratégicas, de infraestrutura e organizacionais do negócio. Dessa forma, todos esses benefícios podem ser analisados e classificados dentro das dimensões da gestão do portfólio de TI (GPTI): benefícios relacionados à infraestrutura de TI, à dimensão transacional, à dimensão informacional e à dimensão estratégica (WEILL e BROADBENT, 1998; WEILL e ARAL, 2006; DOLCl, 2009). Além disso, podese utilizar os Fatores Críticos de Sucesso (FCS), de Somers e Nelson (2001), para melhor entender quais são os principais elementos que devem ser analisados quando as empresas realizam a implementação de sistemas ERP.

Assim, o objetivo desta pesquisa é compreender como os gestores de $\mathrm{TI}$ e de negócio identificam os diferentes benefícios e fatores críticos de sucesso que os sistemas integrados podem proporcionar as suas empresas. Para identificar esses benefícios e fatores críticos, foram realizados estudos de casos em três organizações: um complexo hospitalar, uma rede de farmácias e um supermercado. A partir das informações dos casos, verificou-se que fatores as empresas levam mais em consideração no momento de implementar o ERP. E, além disso, utilizando as dimensões da GPTI, identificou-se uma nova forma de estruturar e analisar os benefícios provenientes do ERP.

O artigo está estruturado da seguinte forma: na seção 2 são apresentados os conceitos de gestão do portfólio de TI (GPTI) e a evolução das dimensões da GPTI, bem como os seus principais conceitos; na seção 
3 são conceitualizados os sistemas ERP e apresentados seus principais benefícios; na seção 4 são apresentados os fatores críticos de sucesso utilizados como base para esta pesquisa; na seção 5 é apresentada a descrição dos casos, enquanto na seção 6 é exposta a discussão dos casos; e por fim na seção 7 são apresentadas as considerações finais da pesquisa.

\section{GESTÃO DE PORTFÓLIO DE TI}

A GPTI é um framework, uma linguagem e uma ferramenta que resulta em uma correlação positiva entre a quantidade investida em TI e o aumento na produtividade, que fornece meios para monitorar e gerenciar os investimentos, de forma que benefícios, custos e riscos de investimentos individuais possam ser avaliados para determinar se eles estão ou não contribuindo significativamente para o desempenho organizacional (SCHNIEDERJANS, HAMAKER e SCHNIEDERJANS, 2004; MAIZLISH e HANDLER, 2005).

Além disso, a GPTI é necessária quando existe a restrição de recursos na empresa, quando o gestor não fica livre para fazer qualquer tipo de investimento de $\mathrm{TI}$ que acredite trazer mais benefícios para a organização (GLIEDMAN, 2002), o que é a realidade da maioria das empresas. Essas limitações ou restrições dizem respeito aos aspectos financeiros, de pessoal, recursos de sistemas e desafios do negócio. Ela fornece uma visão holística de todos os investimentos de TI e auxilia no futuro desses gastos (SYMONS et al., 2005).

Existem diferentes modelos ou frameworks na literatura de TI sobre GPTI (ARCHER e GHASEMZADEH, 1999; MAIZLISH e HANDLER, 2005; ZHENG, 2007), mas com uma visão de portfólio mais generalista e sem identificar o que se conceitua como portfólio. Em outros, o enfoque é na seleção do portfólio de TI (CHO, 2009), ou em regras do planejamento geral do portfólio de TI (KARHADE, SHAW e SUBRAMANYAM, 2009). Nessas pesquisas, foi abordado apenas o tema como parte de uma gestão maior ou a gestão dos investimentos em $\mathrm{Tl}$ como um todo sem focar nas especificidades de cada um. Dessa forma, escolheu-se o modelo inicialmente proposto por Weill e Braodbent (1998) e estudado posteriormente por Aral e Weill (2004), Weill e Aral (2006) e Dolci (2009) onde os investimentos em $\mathrm{Tl}$ são classificados e estruturados em quatro diferentes dimensões de fácil entendimento. A seguir são apresentados a evolução e os principais conceitos e benefícios das dimensões da GPTI.

\subsection{EVOLUÇÃO DAS DIMENSÕES DA GPTI}

Assim como os investimentos financeiros, os investimentos em TI nas empresas podem ser concebidos não como um portfólio financeiro, mas como um portfólio de $\mathrm{TI}$ que contém investimentos com diferentes objetivos gerenciais: dos operacionais aos estratégicos, propiciando diferentes benefícios para as organizações (WEILL e BROADBENT, 1998; 
SCHNIEDERJANS, HAMAKER e SCHNIEDERJANS, 2004; MAIZLISH e HANDLER, 2005; CHO E SHAW, 2009).

O primeiro autor a explorar o tema de portfólio na área de $\mathrm{TI}$ foi Warren McFarlan (1981), que estudou o portfólio de TI mais direcionado para o gerenciamento de projetos de $\mathrm{TI}$ frente a riscos. Posteriormente, Turner e Lucas (1985) apontaram a importância desse tipo de abordagem para analisar os investimentos em TI sem denominá-la como GPTI. Entretanto, pode-se considerar que o trabalho de Turner e Lucas (1985) é o marco inicial que apresenta dimensões para gestão dos investimentos em TI e que auxiliou nas pesquisas preliminares de pesquisadores no tema GPTI. No trabalho desses autores, foi realizada a separação de todos os investimentos em $\mathrm{TI}$ de acordo com os objetivos organizacionais que eles ajudam a alcançar. Esses objetivos gerenciais foram especificados, derivados e estendidos da pesquisas de Turner e Lucas (1985) por Weill (1992), passando a ser chamados de: (i) TI transacional; (ii) TI estratégica; e (iii) TI informacional. Esses objetivos foram chamados de dimensões (WEILL e BROADBENT, 1998). Uma quarta dimensão foi incluída posteriormente: a infraestrutura de $\mathrm{TI}$ pesquisada por diversos autores (BROADBENT e WEILL, 1997; BROADBENT, WEILL e NEO, 1999; WEILL, SUBRAMANI e BROADBENT, 2002).

\subsection{AS DIMENSÕES DA GPTI}

Após a identificação e as origens de todas as dimensões, são apresentados os investimentos de TI como um portfólio de TI com quatro dimensões (WEILL e BROADBENT, 1998). A partir dos estudos de Weill e Broadbent (1998); Aral e Weill (2004) e Weill e Aral (2006), posteriormente foi realizada uma exploração das dimensões com empresas e executivos de $\mathrm{Tl}$ brasileiros (DOLCl, 2009). As dimensões e os seus benefícios são definidos, caracterizados e compostos, a seguir.

A dimensão de infraestrutura de TI é a base do portfólio, proporcionando a estrutura para os serviços de TI compartilhados (humanos e técnicos como, por exemplo, computadores pessoais, equipamentos de comunicação, servidores, rede, laptops, base de dados, help desk, etc.). Representam investimentos realizados para prover uma base flexível para as iniciativas de negócios futuros. Os benefícios com os investimentos que compõem essa dimensão estão ilustrados na Figura 1.

A dimensão transacional representa gastos e investimentos para suportar as tarefas diárias e operacionais da organização, tais como ordens de pedidos, controle de inventário, gestão de caixa, contas a receber, contas a pagar, relatórios de produção, e outros processamentos cotidianos da empresa. Os benefícios com os investimentos que compõem essa dimensão também estão ilustrados na Figura 1.

A dimensão informacional representa gastos e investimentos visando a melhorar a disponibilidade de informações para o gerenciamento e controle da empresa, suportando a tomada de decisão, planejamento, comunicação, contabilidade e análises. Essas informações provêm de resumos obtidos de sistemas componentes da dimensão transacional. Os 
benefícios que compõem essa dimensão também estão ilustrados na Figura 1.

A dimensão estratégica é composta por investimentos para reposicionar e manter a empresa no mercado. Os investimentos nessa dimensão tipicamente alteram a natureza dos serviços de TI e os processos organizacionais na indústria. Dada a natureza dinâmica da $\mathrm{Tl}$, muitos investimentos considerados estratégicos em um dado momento e espaço, deixam de sê-lo com o passar do tempo, em um processo de comoditização. É o caso, por exemplo, das caixas eletrônicas de banco. Quando de sua introdução, alteraram os serviços entregues para os clientes bancários, e garantiram vantagens para os primeiros bancos que as adotaram. Hoje, entretanto, representam investimentos transacionais (e não mais estratégicos), na medida em que se tornaram universalmente adotadas (WEILL e BROADBENT, 1998).

A Figura 1 apresenta as dimensões do portfólio de investimentos em TI segundo uma estrutura hierárquica, assim como os benefícios associados a cada uma delas.

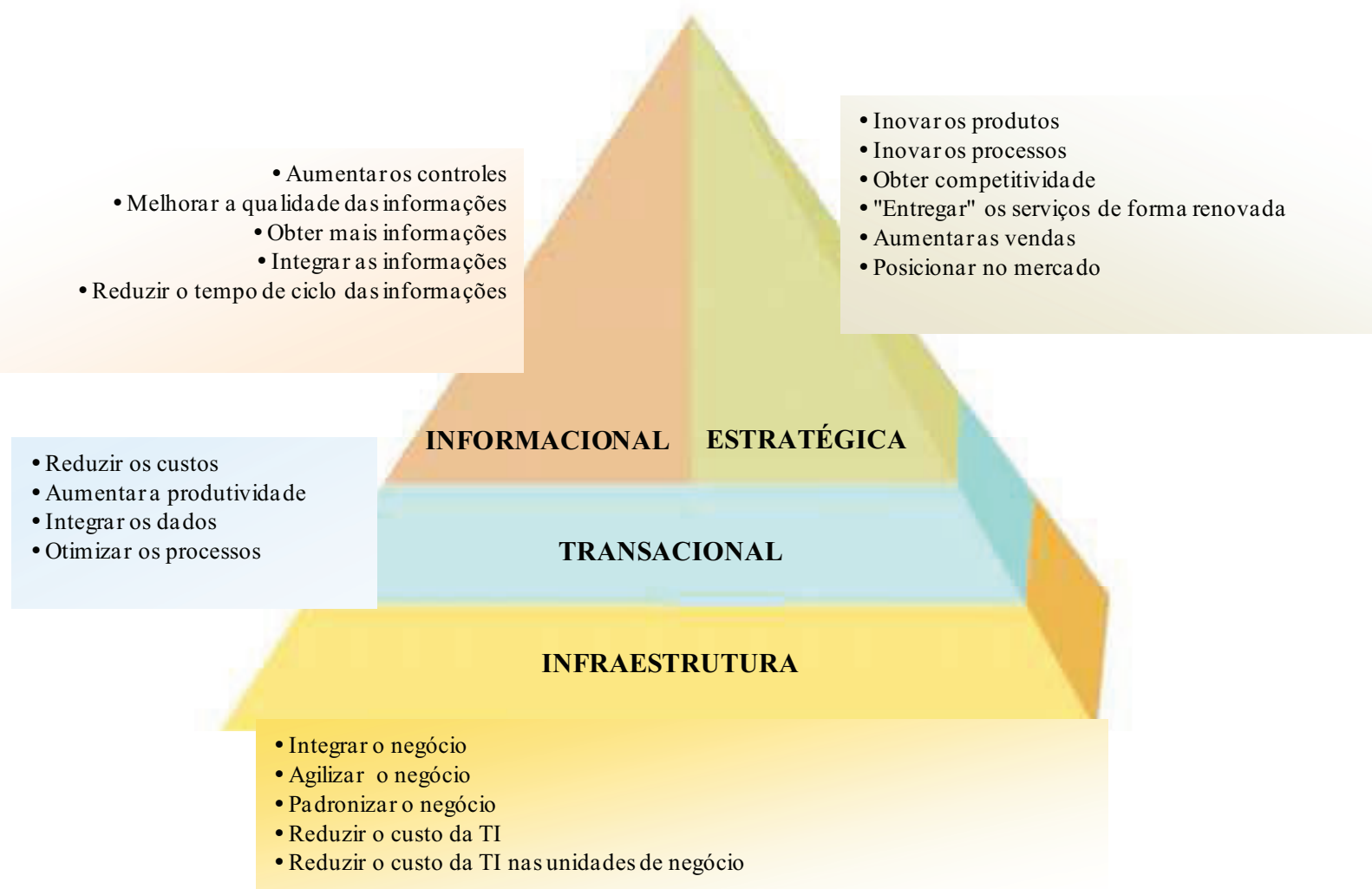

Figura 1. Dimensões da gestão do portfólio de TI e benefícios dos investimentos em TI Fonte: Dolci (2009)

\section{ERP}

Os sistemas ERP são sistemas integrados de negócio que são utilizados para automatizar o fluxo de materiais, informações e recursos financeiros entre todas as funções dentro da empresa utilizando uma base de dados comum (SU e YANG, 2010). Koh, Gunasekaran e Rajkumar 
(2008) apontam que, no futuro, as empresas cada vez mais estão sendo dirigidas pelas competências centrais e com investimentos menores nas demais atividades.

Os sistemas ERP são considerados de vital importância para as grandes empresas, fazendo parte do negócio, onde esses sistemas são a combinação de processos de negócio e TI (SU e YANG, 2010). Devido aos altos investimentos em TI realizados pelas organizações e à importância e impacto que a implementação dos sistemas ERP causam nas empresas é necessário analisar os benefícios que esses sistemas proporcionam. Existem diversos casos de problemas relacionados com a implementação de sistemas ERP, podendo-se observar que de $20 \%$ a $35 \%$ falham e que o resultado de até $80 \%$ dos projetos são questionáveis por excederem o tempo e/ou orçamento propostos (MEDEIROS JUNIOR, 2009). Então, novas maneiras de analisar os benefícios dos sistemas, podem auxiliar as organizações a obter mais sucesso nesse processo. Dessa forma, foram utilizados os benefícios das quatro dimensões da GPTI, realizando associações com os benefícios dos sistemas ERP para melhor identificar e visualizar os benefícios dos sistemas ERP.

Na década de 90, Davenport (1998) apontava que a literatura de ERP era focada em pesquisas sobre implementação e os desafios da implementação dos projetos por si só. Recentemente, diferentes correntes de pesquisa sobre ERP são observadas na literatura (SU e YANG, 2010). Uma dessas correntes está relacionada aos benefícios que os sistemas ERP proporcionam para toda a empresa. Uma justificativa para realizar investimentos em sistemas ERP bem feita ajuda a identificar potenciais benefícios que podem florescer com a implementação dos sistemas ERP (MALHOTRA e TEMPONI, 2010). Esses potenciais benefícios mais tarde se tornam determinantes para o desempenho do negócio (CHEN, 2001).

Diversos autores afirmam que a implementação de sistemas ERP proporciona diferentes benefícios para as organizações. A seguir, são apresentados os benefícios encontrados tanto na literatura acadêmica, quanto na comercial. No final da seção é apresentada uma tabela relacionando os benefícios dos sistemas ERP com as quatro dimensões da GPTI. Davenport (1998) propõe que a implementação de sistemas ERP proporciona muitos benefícios para as organizações, incluindo a redução do tempo de ciclo das informações, a melhora do fluxo de informações, a geração rápida de informações financeiras e integração de informações (financeiras, contábeis, de recursos humanos, da cadeia de suprimento e de clientes) ao longo da empresa e o auxílio no desenvolvimento de novas estratégias organizacionais.

Turban, McLean e Wetherbe (2004) afirmam que o sistema ERP proporciona soluções que beneficiam e melhoram a eficiência, qualidade das informações e produtividade da empresa, elevando o resultado e também a satisfação dos clientes. Na busca dos benefícios oferecidos por um sistema de ERP, as empresas ao decidirem por sua adoção esperam manter sua capacidade de sobrevivência no longo prazo. Já para Laudon e Laudon (2004) esses benefícios podem ser classificados em tangíveis e 
não tangíveis, sendo que, quanto maior for o número dos benefícios intangíveis, mais difícil é a sua mensuração.

Berchet e Habchi (2005) consideram que o ERP reflete em estratégias de negócio inovadoras, envolvendo melhorias nos processos de negócio, implementação de melhores práticas e integração intra-organizacional. Além disso, os sistemas ERP são considerados como uma ferramenta poderosa e robusta para as reais mudanças na indústria (BERCHET e HABCHI, 2005). Para Mazutti, Maçada e Rios (2005), os sistemas ERP ajudam as empresas a planejar e alocar seus recursos, enquanto aumentam o controle e a visibilidade ao longo da empresa, além de auxiliar no aumento da produtividade e diminuição dos custos.

Os benefícios percebidos dos sistemas ERP, segundo Holsapple e Sena (2005), são: melhoria dos processos de negócio, diminuição de custos, resolução de problemas de sistemas legados, auxílio no aumento da empresa, diminuição do pessoal, eficiência operacional, redução de treinamento e pessoal de suporte, melhoria na gestão de estoques, suporte no aumento das vendas, posicionamento frente à demanda, melhoria na confiabilidade do sistema, auxílio na integração dos dados e melhoria na agilidade do negócio.

Para Souza e Zwicker (2006) os sistemas ERP contribuem para o negócio na melhoria do fluxo de informações entre departamentos, na melhoria do fluxo de informações na cadeia de suprimentos, no incremento do desempenho operacional, na melhoria da performance de funcionários, na redução de retrabalho, no aumento da facilidade de uso dos sistemas, na redução das despesas de TI e no aumento do grau de terceirização da TI. Cabe ressaltar, que os objetivos dos sistemas ERP encontrados no estudo desses autores são definidos principalmente pela alta direção e a área de TI, devendo-se observar os benefícios dos sistemas ERP. Para Wang et al. (2008) a implementação do sistema ERP é considerada um sucesso quando se consegue atingir os seguintes potenciais benefícios: redução de pessoal, diminuição dos custos da Tl, melhor controle de estoques e melhora da gestão financeira da empresa.

Quando um sistema ERP está completamente finalizado em uma organização, pode-se esperar o rendimento de muitos benefícios (SU e YANG, 2010): redução do tempo de ciclo das informações, transações mais rápidas e melhor gestão financeira. Uma vez que os potenciais benefícios são grandes, muitas organizações estão dispostas a empreender o difícil processo de conversão de tudo o que usam atualmente, devido aos altos custos e os riscos envolvidos, para um sistema de ERP (CHEN e LIN, 2009; SU e YANG, 2010).

Em relação aos benefícios dos sistemas ERP na literatura comercial, foi realizada a busca nos dois maiores fornecedores no país (LMBARROS, 2009): SAP e Oracle. De acordo com a SAP (www.sap.com.br) os benefícios são: maior alinhamento entre estratégias e operações; maior produtividade e visibilidade; maior flexibilidade, resultando em menores custos; suporte aos requisitos da indústria, em constante mudança; riscos reduzidos; melhor gestão financeira e governança corporativa; e, otimização dos 
gastos de TI. Para a Oracle (www.oracle.com.br) os benefícios dos sistemas ERP estão relacionados com a padronização, documentação, eficiência, integração, flexibilidade, organização e segurança.

A partir dos diferentes benefícios levantados na literatura acadêmica e comercial, foi realizada uma análise cruzada com os benefícios das dimensões da GPTI, onde cada um dos benefícios das dimensões foi relacionado com os encontrados na literatura referente aos sistemas ERP. $\mathrm{Na}$ Tabela 1 são apresentados esses relacionamentos.

\section{FATORES CRÍTICOS DE SUCESSO}

Os fatores críticos de sucesso (FCS) podem ser vistos como uma forma de auxiliar os gestores de $\mathrm{Tl}$ e de negócio para melhorias nos processos organizacionais, cujo efeito é muito mais rico se visto dentro de um determinado contexto levando em conta cada uma das etapas do processo de execução (SOMERS e NELSON, 2001). Esse processo de execução consiste em seis fases distintas: iniciação, adoção, adaptação, aceitação, rotinização e infusão (COOPER E ZMUD, 1990).

Dentro dessas fases existem diferentes fatores que afetam o processo de implementação de um sistema ERP e influenciam seu sucesso ou fracasso. Os fatores mais importantes são (SOMERS e NELSON, 2001): suporte da alta gerência e envolvimento, a necessidade de um campeão do projeto, a formação de utilizadores, a competência tecnológica, o processo de delimitação, o planejamento, gestão de mudanças e gerenciamento de projeto. No contexto da implementação do ERP, problemas adicionais incluem a necessidade de reestruturar os processos de negócios antes da sua implementação, a necessidade de comunicar de forma eficaz e definir as expectativas adequadas, o uso de uma equipe de negócios equilibrada e evitar, quando possível, a customização do sistema (SUMNER, 1999).

No trabalho de Somers e Nelson (2001) foi proposta uma lista com 22 fatores críticos de sucesso (FCS) associados com a implementação de sistemas resultantes de um processo que envolve a identificação e síntese dos requisitos críticos para a implementação de diferentes sistemas, incluindo o ERP. Cada um desses fatores pode ser analisado dentro das fases mencionadas anteriormente (iniciação, adoção, adaptação, aceitação, rotinização e infusão). A lista com todos os fatores em ordem de importância é apresentada no Quadro 1. 
Tabela 1. Cruzamento dos benefícios das dimensões da GPTI com os benefícios dos sistemas ERP

\begin{tabular}{|c|c|c|c|c|}
\hline Dim. & \multicolumn{2}{|c|}{ Benefícios das Dimensões da GPTI } & \multicolumn{2}{|c|}{ Benefícios dos Sistemas ERP } \\
\hline \multirow{4}{*}{ 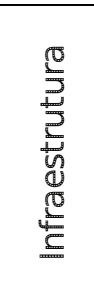 } & Integrar o negócio & \multirow{15}{*}{$\begin{array}{l}\text { Weill e } \\
\text { Broadbent } \\
\text { (1998) }\end{array}$} & Integrar o negócio e integrar as partes da organização. & Oracle; Berchet e Habchi (2005) \\
\hline & Agilizar o negócio & & Melhorar a agilidade do negócio. & Holsapple e Sena (2005) \\
\hline & Padronizar o negócio & & Padronizar. & Oracle \\
\hline & $\begin{array}{l}\text { Reduzir o custo da TI } \\
\text { nas unidades de } \\
\text { negócio }\end{array}$ & & $\begin{array}{l}\text { Otimizar os gastos em } \mathrm{Tl} \text { e reduzir as despesas e custos de } \\
\text { TI. }\end{array}$ & $\begin{array}{l}\text { SAP; Souza e Zwicker (2006); Wang et al } \\
\text { (2008) }\end{array}$ \\
\hline & Reduzir os custos & & $\begin{array}{l}\text { Diminuir os custos; diminuir custos de treinamento e de } \\
\text { pessoal }\end{array}$ & $\begin{array}{l}\text { SAP; Mazutti, Maçada e Rios (2005); } \\
\text { Holsapple e Sena (2005); Wang et al (2008) }\end{array}$ \\
\hline & Integrar os dados & & Auxiliar na integração dos dados. & Holsapple e Sena (2005) \\
\hline & Otimizar os processos & & Melhorar os processos de negócio. & $\begin{array}{l}\text { Berchet e Habchi (2005); Holsapple e Sena } \\
\text { (2005) }\end{array}$ \\
\hline \multirow{2}{*}{ 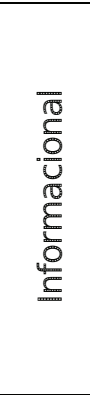 } & Aumentar os controles & & Aumentar o controle e visibilidade ao longo da empresa. & Mazutti, Maçada e Rios (2005) \\
\hline & $\begin{array}{l}\text { Reduzir o tempo de } \\
\text { ciclo das informações }\end{array}$ & & Reduzir o tempo de ciclo das informações. & Davenport (1998); Su e Yang (2010) \\
\hline \multirow{6}{*}{ 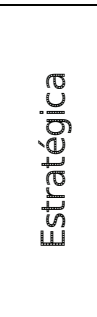 } & Inovar os produtos & & Propiciar estratégias de negócio inovadoras. & Berchet e Habchi (2005) \\
\hline & Inovar os processos & & Propiciar estratégias de negócio inovadoras. & Berchet e Habchi (2005) \\
\hline & Obter competitividade & & $\begin{array}{l}\text { Auxiliar no desenvolvimento de novas estratégias } \\
\text { organizacionais. }\end{array}$ & Davenport (1998) \\
\hline & $\begin{array}{l}\text { "Entregar" os serviços } \\
\text { de forma renovada }\end{array}$ & & Suportar os requisitos da indústria, em constante mudança. & SAP \\
\hline & Aumentar as vendas & & Suportar o aumento das vendas. & Holsapple e Sena (2005) \\
\hline & Posicionar no mercado & & Posicionar frente à demanda. & Holsapple e Sena (2005) \\
\hline
\end{tabular}

Fonte: elaborado pelos autores.

(2) Revista Eletrônica de Sistemas de Informação, v. 9, n. 1, artigo 4 


\begin{tabular}{|l|l|}
\hline 1. Suporte da alta gerência & 12. Recursos dedicados \\
\hline 2. Competência do time de projeto & 13. Uso do comitê de direção \\
\hline 3. Cooperação Interdepartamental & 14. Treinamento do usuário no software \\
\hline 4. Clareza dos objetivos e metas & $\begin{array}{l}\text { 15. Educação em novos processos de } \\
\text { negócios }\end{array}$ \\
\hline 5. Gestão de projetos & 16. Reengenharia dos processos de negócio \\
\hline 6. Comunicação interdepartamental & 17. Customização mínima \\
\hline 7. Gestão das expectativas & 18. Escolha da arquitetura \\
\hline 8. Líder de projeto & 19. Gestão da mudança \\
\hline 9. Suporte de vendas & 20. Parceria com o fabricante \\
\hline 10. Seleção cuidadosa do pacote & 21. Uso de ferramentas dos vendedores \\
\hline 11. Análise e conversão dos dados & 22. Uso de consultores \\
\hline
\end{tabular}

Quadro 1. Lista dos 22 FCS.

Fonte: adaptado de Somers e Nelson, 2001

Como mencionado anteriormente, cada um dos FCS pode ser analisado dentro de cada uma das fases da implementação de um determinado sistema, encontrando os mais importantes. Pode-se identificar os FCS em conjunto com os benefícios que os sistemas ERP proporcionam para gerar mais informações sobre o processo de implementação e auxiliar na tomada de decisão.

\section{MÉTODO}

A partir da definição dos benefícios das dimensões da GPTI e a conceituação dos fatores críticos de sucesso, foi realizado um estudo de caso múltiplo em três diferentes empresas, com o objetivo de compreender como os gestores analisam os benefícios e os fatores críticos de sucesso no momento de realizar investimentos em sistemas integrados. As empresas analisadas são de grande porte, com receita superior a 300 milhões de reais por ano e grandes investimentos em $\mathrm{Tl}$, atuando nos setores hospitalar, atacadista e farmacêutico.

Os gestores de TI e de negócio foram entrevistados, utilizando-se um protocolo de estudo caso, que se destina a orientar o pesquisador ao realizar a coleta de dados em um estudo de caso (YIN, 2005). O protocolo relaciona as atividades a serem realizadas durante a pesquisa, apontando os procedimentos a serem adotados, auxiliando a manter os mesmos procedimentos em um estudo de caso múltiplo (BENBASAT; GOLDSTEIN e MEAD, 1987; DUBÉ e PARÉ, 2003; OLIVERIA, MAÇADA e GOLDONI, 2009). As atividades desse protocolo estão relacionadas à coleta de algumas informações como: dados da empresa, faturamento da empresa e os investimentos em ERP.

Além disso, o protocolo contém o roteiro de entrevista semiestruturado baseado nas dimensões da GPTI (DOLCl, 2009), nos fatores críticos de sucesso (SOMERS e NELSON, 2001) analisados no processo de implementação do ERP em seis distintas fases: iniciação, adoção, adaptação, aceitação, rotinização e infusão (COOPER E ZMUD, 1990). O protocolo da pesquisa foi validado no desenvolvimento do estudo de caso piloto. Esta etapa da investigação aumentou a confiabilidade, pois forneceu informa- 
ções que permitiram que a pesquisa fosse repetida nas mesmas condições e que atingisse os mesmos resultados (RIEGE, 2003; YIN, 2005).

As entrevistas em profundidade foram realizadas pessoalmente e duraram em média duas horas e vinte minutos. Foram levantadas informações referentes aos investimentos em ERP, sendo analisada a implementação de um sistema ERP utilizando as dimensões da GPTI e os fatores críticos de sucesso. Nas empresas pesquisadas, os recursos financeiros destinados aos investimentos em ERP ficaram entre 650 mil reais e um milhão de reais. Essas empresas estavam na fase de seleção de um sistema integrado ou na substituição do sistema utilizado atualmente. Além disso, os entrevistados possuem grande conhecimento da área de $\mathrm{Tl}$, conhecem os processos de negócio das empresas e possuem grande familiaridade com o ERP.

Todas as entrevistas foram gravadas e posteriormente transcritas. As entrevistas foram analisadas utilizando a análise de conteúdo, realizando a descrição das decisões referente a cada um dos sistemas ERP, onde os gestores apresentaram os motivos que levaram a decidir por implementar os sistemas ERP. Foram analisadas a escolha e a seleção do fornecedor, identificando os benefícios dos sistemas ERP, bem como os principais FCS.

A partir dos benefícios percebidos pelos gestores, baseado na literatura levantada, realizou-se a estruturação dos benefícios do ERP dentro de cada uma das dimensões da GPTI: infraestrutura, transacional, informacional e estratégica. Foram abordados todos os benefícios dos sistemas ERP identificados pelo entrevistado e analisados utilizando os benefícios das quatro dimensões. Nos fatores críticos de sucesso, foram elencados quais eram os fatores mais importantes dentro de cada uma das fases de iniciação, adoção, adaptação, aceitação, rotinização e infusão.

Sendo solicitado pelos respondentes, os nomes das empresas foram mantidos em sigilo, sendo tratadas apenas pelo setor a que elas pertencem.

\section{DESCRIÇÃO DOS CASOS}

Nesta seção é apresentada uma breve descrição dos casos, com as características das empresas e como foram realizadas as decisões de investimentos nos sistemas ERP.

\section{Organização 1 - Complexo Hospitalar}

A organização 1 é um grande complexo hospitalar brasileiro, composto por sete diferentes hospitais. O complexo faturou mais de 300 milhões de reais em 2008, realizando mais de 700.000 consultas, 40.000 internações e 60.000 cirurgias. Mais de 20.000 pessoas circulam pelo conjunto de hospitais por dia, sendo atendidos por quase 6.000 funcionários.

A implementação do ERP foi motivada por algumas dificuldades que a empresa vem enfrentando nos últimos anos, segundo os gestores de $\mathrm{TI}$ e negócio. Esses problemas foram divididos em financeiros e gerenciais. Os

(2) Revista Eletrônica de Sistemas de Informação, v. 9, n. 1, artigo 4 DOI: 10.5329/RESI.2010.0901004 
financeiros são: glosas ${ }^{2}$ nas contas hospitalares SUS, por não comprovarem o uso do material ou serviço; glosas nas contas hospitalares Convênio, por não comprovarem o uso do material ou serviço; inadimplência de clientes particulares; perda financeira por não lançamento de itens na conta hospitalar; perda de registro dos atendimentos prestados; e perda de estoque por ter baixo controle e furtos internos. Os gerenciais foram: o fato de o departamento de TI não ser estratégico para a empresa; corrupção do setor e compra, favorecendo alguns fornecedores em troca de vantagens pessoais; custo de retrabalho alto pela baixa integração dos sistemas (nove sistemas identificados dentro da instituição); e, perda de informações importantes devido ao tráfego interno de documentos.

A partir desses problemas, foi determinada pela direção do complexo a aquisição de um sistema ERP identificando os benefícios que o sistema iria proporcionar, utilizando as dimensões da GTPI e os fatores críticos de sucesso. A empresa possuía um valor máximo de investimento de $R \$$ $1.000 .000,00$. O fornecedor do sistema e demais serviços deveria respeitar alguns critérios de seleção avaliados pelos gestores por notas de 1 a 5 : tipo de produto; tipo de licença; número de usuários recomendados; público alvo; plataforma; arquitetura modular; apoia a conformidade regulatória; suporte a SOA; e uso de estrutura de portal. Além desses critérios, foi analisada a aderência aos processos da empresa e feito um benchmark dos sistemas utilizados na concorrência. Com essas informações, onze diferentes empresas no mercado se adequaram aos critérios estabelecidos. Uma empresa apresentou média mais alta e foi escolhida como a empresa a ser contratada.

Além dessa análise de seleção da empresa, foi realizada pelos gestores a análise dos benefícios que o ERP podia proporcionar para a empresa utilizando as dimensões da GPTI e os fatores críticos de sucesso. Os benefícios de infraestrutura de TI que o ERP poderia proporcionar para a empresa, percebidos pelos gestores, foram: construção de flexibilidade do negócio para mudanças atuais e futuras; redução de custo de Tl; e melhoria da capacidade de infraestrutura de TI. Os benefícios transacionais foram: redução dos custos operacionais, redução dos custos de comunicação, eliminação da necessidade do aumento da força de trabalho, aumento do retorno nos ativos financeiros, aumento da produtividade dos funcionários e economias na gestão da cadeira de suprimentos. Os informacionais foram: acesso facilitado e mais rápido à informação; melhor informação gerencial para o planejamento estratégico, aumento da precisão da informação e provimento de informação em vários formatos. Por fim, os estratégicos foram: alinhamento da estratégia de TI com a de negócio, criação de vantagem competitiva, respostas mais rápidas às mudanças e relações melhores com os clientes.

2 Glosa é a recusa parcial ou total de uma fatura, pela operadora de plano de saúde, por considerar sua cobrança indevida, por erro ou omissão de alguma informação nas fichas de atendimento ou pedido de pagamento. 
A distribuição em porcentagens dos benefícios em cada uma das dimensões foi: $20 \%$ na infraestrutura, $30 \%$ na transacional, $40 \%$ na informacional e $10 \%$ na estratégica. Os fatores críticos de sucesso, segundo os respondentes, para a implementação do ERP, em ordem de importância, em cada uma das fases (SOMERS e NELSON, 2001) são apresentadas no Quadro 2.

\begin{tabular}{|c|c|c|c|}
\hline & Iniciação & Adoção & Adaptação \\
\hline 1 & Escolha da arquitetura & Suporte da alta gerência & $\begin{array}{c}\text { Comunicação } \\
\text { interdepartamental }\end{array}$ \\
\hline 2 & $\begin{array}{c}\text { Clareza dos objetivos e } \\
\text { metas }\end{array}$ & $\begin{array}{c}\text { Competência do time de } \\
\text { projeto }\end{array}$ & Gestão da mudança \\
\hline 3 & Uso do comitê de direção & Parceria com o fabricante & $\begin{array}{c}\text { Competência do time } \\
\text { de projeto }\end{array}$ \\
\hline & Aceitação & Rotina & Infusão \\
\hline 1 & $\begin{array}{c}\text { Comunicação } \\
\text { interdepartamental }\end{array}$ & $\begin{array}{c}\text { Comunicação } \\
\text { interdepartamental }\end{array}$ & $\begin{array}{c}\text { Comunicação } \\
\text { interdepartamental }\end{array}$ \\
\hline 2 & $\begin{array}{c}\text { Clareza dos objetivos e } \\
\text { metas }\end{array}$ & Suporte da alta gerência & $\begin{array}{c}\text { Cooperação } \\
\text { Interdepartamental }\end{array}$ \\
\hline 3 & Suporte da alta gerência & Suporte de vendas & $\begin{array}{c}\text { Suporte da alta } \\
\text { gerência }\end{array}$ \\
\hline
\end{tabular}

Quadro 2. FCS da organização1

Fonte: elaborado pelos autores.

\section{Organização 2 - Rede de Farmácias}

A organização 2 é uma rede de farmácias com mais de 240 lojas em 89 municípios do Rio Grande do Sul e Santa Catarina, com um faturamento em 2008 de aproximadamente 600 milhões de reais. A rede é líder no segmento no RS, e é, atualmente, uma das maiores redes de farmácias do Brasil.

Atualmente, a empresa está planejando substituir o ERP que possui por outro, com o objetivo, segundo os respondentes, de aumentar a receita, resolver questões táticas, realizar um upgrade tecnológico, reduzir a relação custo/melhoria da eficiência, melhorar o processo de decisão dos executivos e melhorar a exatidão e a disponibilidade da informação.

Dessa forma, foi analisado o novo ERP a ser adquirido identificando os benefícios que ele pode proporcionar, usando como referência as dimensões da GPTI e os FCS. A empresa possuía um recurso para ser investido de aproximadamente $\mathrm{R} \$ 650.000,00$, mas o fornecedor escolhido deveria respeitar alguns critérios de seleção: critérios técnicos, funcionalidade, referência do fornecedor, implantabilidade, riscos, flexibilidade, custo, serviços e suporte, confiabilidade do sistema, facilidades de customização, ajuste à estrutura, consultoria e implementação, tempo de implementação, métodos de implementação do software, domínio do fornecedor, posição do fornecedor no mercado, compatibilidade com outros sistema, facilidade de uso, visão do fornecedor, escalabilidade para crescimento e segurança. Três diferentes empresas no mercado se adequaram aos critérios estabelecidos e foram profundamente analisadas. Uma empresa apresentou a melhor relação custo/benefício e foi escolhida como a empresa a ser contratada. 
Na etapa seguinte foram identificados os benefícios que o ERP poderia proporcionar para a empresa, segundo o entrevistado, utilizando as dimensões da GPTI e aos fatores críticos de sucesso. Os benefícios de infraestrutura de $\mathrm{TI}$ percebidos pelos gestores foram: padronização do ambiente de $\mathrm{Tl}$; agilidade na resposta das informações e flexibilidade do negócio. Os benefícios transacionais foram: criação de inteligência; otimização da logística, redução de custos, otimização dos processos e redução de custos. Os benefícios informacionais foram: maior controle, qualidade nas informações e integração organizacional. Por fim, os benefícios estratégicos foram: inovação de mercado; inovação nos processos; alavancagem das vendas e melhoria da posição perante o mercado.

A distribuição em porcentagens dos benefícios em cada uma das dimensões foi: $20 \%$ na infraestrutura, $40 \%$ na transacional, $30 \%$ na informacional e $10 \%$ na estratégica. Os fatores críticos de sucesso, segundo os respondentes, para a implementação do ERP são apresentadas no Quadro 3, em ordem de importância em cada uma das fases (SOMERS e NELSON, 2001).

\begin{tabular}{|c|c|c|c|}
\hline & Iniciação & Adoção & Adaptação \\
\hline 1 & $\begin{array}{c}\text { clareza dos objetivos e } \\
\text { metas }\end{array}$ & suporte da alta gerencia & $\begin{array}{l}\text { educação em novos } \\
\text { processos de negócios }\end{array}$ \\
\hline 2 & escolha da arquitetura & gestão das expectativas & gestão da mudança \\
\hline 3 & $\begin{array}{c}\text { cooperação } \\
\text { interdepartamental }\end{array}$ & parceria com o fabricante & $\begin{array}{c}\text { análise e conversão dos } \\
\text { dados }\end{array}$ \\
\hline & Aceitação & Rotina & Infusão \\
\hline 1 & gestão das expectativas & parceria com o fabricante & suporte da alta gerencia \\
\hline 2 & suporte da alta gerencia & $\begin{array}{c}\text { análise e conversão dos } \\
\text { dados }\end{array}$ & $\begin{array}{c}\text { clareza dos objetivos e } \\
\text { metas }\end{array}$ \\
\hline 3 & $\begin{array}{c}\text { cooperação } \\
\text { interdepartamental }\end{array}$ & $\begin{array}{c}\text { cooperação } \\
\text { interdepartamental }\end{array}$ & $\begin{array}{c}\text { cooperação } \\
\text { interdepartamental }\end{array}$ \\
\hline
\end{tabular}

Quadro 3. FCS da organização 2

Fonte: elaborado pelos autores.

\section{Organização 3 - Supermercado}

A organização 3 é um grupo de diferentes empresas, onde um dos negócios são os supermercados com atuação no Brasil e na Europa. Atualmente o grupo está realizando uma série de investimentos e atualizações em suas empresas no Brasil, dentre as quais um supermercado do grupo foi analisado. O faturamento da empresa não foi disponibilizado pelos entrevistados.

A empresa está realizando um projeto de substituição do ERP atual (home made) por uma solução robusta e integrada que atenda as necessidades de negócio, reduzindo a carga sobre a estrutura de custos da empresa e mantendo-se dentro da expectativa de investimento de $\mathrm{R} \$ 750.000,00$.

O novo ERP a ser adquirido foi analisado utilizando como referência as dimensões da GPTI e os FCS. Foi analisada a aderência dos sistemas ERP aos processos corporativos divididos em finanças (bancos e pagamentos; gestão de custos; contabilidade geral), CRM (gestão de atividades; gestão de serviços), SCM (gestão de vendas, gestão de compras), marketing (geren- 
ciamento de campanhas; gerenciamento de preços), BI (relatórios analíticos; ferramentas de análise), vendas (previsão e análise de vendas; gestão das lojas) e recursos humanos (gestão de pessoal; gestão de recrutamento e seleção). Três fornecedores foram analisados segundo esses critérios de aderência, classificando-se cada um dos processos como: aderência total, parcial ou sem aderência, além de analisar as vantagens e desvantagens de cada fornecedor. A empresa que obteve mais processos como aderência total e que estava dentro dos limites financeiros foi escolhida.

$\mathrm{Na}$ etapa seguinte foram identificados os benefícios que o ERP poderia proporcionar para a empresa, segundo o entrevistado, utilizando as dimensões da GPTI e aos fatores críticos de sucesso. Os benefícios de infraestrutura de TI que o ERP poderia proporcionar para a empresa, percebidos pelos gestores, foram: a padronização do negócio, através da eliminação de aplicativos legados e a redução do custo com $\mathrm{Tl}$, com a redução de atendimentos no service desk, redução de pessoal para manutenção de sistemas e centralização de contratos de suporte. Os benefícios transacionais foram: redução de custos operacionais, como retrabalho e eliminação de redundâncias, diminuiç̧ão de erro nos processos; e aumento de produtividade e da satisfação dos usuários, através da simplificação e automatização de processos e otimização do fluxo das informações. Os benefícios informacionais foram: otimização do processo de tomada de decisão, possibilitando maior confiabilidade das informações e suporte avançado de business intelligence (BI); melhor controle sobre processos financeiros; e, melhor planejamento da cadeia de suprimentos. E por fim, os benefícios estratégicos, percebidos pelos gestores foram: redução do tempo de resposta ao mercado com a antecipação das necessidades do cliente; aumento das vendas, através de campanhas direcionadas; e melhor posicionamento no mercado através da segmentação de clientes.

A distribuição em porcentagens dos benefícios em cada uma das dimensões foi: $20 \%$ na infraestrutura, $40 \%$ na transacional, $30 \%$ na informacional e $10 \%$ na estratégica. Os fatores críticos de sucesso, segundo os respondentes, para a implementação do ERP são apresentadas no Quadro 4, em ordem de importância em cada uma das fases (SOMERS e NELSON, 2001).

\begin{tabular}{|c|c|c|c|}
\hline & Iniciação & Adoção & Adaptação \\
\hline 1 & uso do comitê de direção & $\begin{array}{c}\text { competência do time de } \\
\text { projeto }\end{array}$ & $\begin{array}{c}\text { análise e conversão dos } \\
\text { dados }\end{array}$ \\
\hline 2 & escolha da arquitetura & $\begin{array}{c}\text { reengenharia de } \\
\text { processos de negócio }\end{array}$ & gestão da mudança \\
\hline 3 & uso de consultores & $\begin{array}{c}\text { customização mínima } \\
\text { procengenharia de }\end{array}$ & $\begin{array}{c}\text { Infusão } \\
\text { Rotina }\end{array}$ \\
\hline 1 & Aceitação & $\begin{array}{c}\text { comunicação } \\
\text { suporterdepartamental }\end{array}$ & suporte da alta gerência gerência \\
\hline 2 & líder de projeto & $\begin{array}{c}\text { educação em novos } \\
\text { processos de negócios }\end{array}$ & $\begin{array}{c}\text { comunicação } \\
\text { interdepartamental }\end{array}$ \\
\hline 3 & gestão das expectativas & suporte de vendas & gestão das expectativas \\
\hline
\end{tabular}

Quadro 4. FCS da organização 3

Fonte: elaborado pelos autores.

(2) Revista Eletrônica de Sistemas de Informação, v. 9, n. 1, artigo 4 DOI: 10.5329/RESI.2010.0901004 


\section{DISCUSSÃO DOS RESULTADOS}

Está seção apresenta uma análise dos principais resultados que foram encontrados nos três casos analisados. Todas as empresas estavam em processo de aquisição de sistemas ERP. Uma delas estava adquirindo um novo ERP (organização 1 - hospital) e as demais estavam substituindo o sistema que já utilizavam (organização 2 - farmácia e organização 3 - supermercado).

Nos três casos analisados, as empresas possuíam processos formais de escolha e seleção dos potenciais fornecedores, com a utilização de notas e/ou classificações de acordo com critérios pré-definidos. Pôde-se observar que cada uma das empresas realizou diferentes formas de análises dos fornecedores com diferentes atributos considerados. Dessa forma, analisar os benefícios e os FCS pode auxiliar os gestores de TI e de negócio na atividade de selecionar o melhor ERP no mercado, de acordo com as necessidades e objetivos da organização.

Em relação aos benefícios que os sistemas ERP proporcionam utilizando as dimensões da GPTI, pôde-se observar algumas particularidades. Os benefícios de infraestrutura de TI estão relacionados às capacidades que a TI tem ou precisa ter para suportar as atividades das empresas frente a mudanças atuais e futuras. Benefícios como agilidade, flexibilidade, redução de custos e diminuição de sistemas legados, na opinião dos gestores. Os benefícios transacionais foram relacionados à: redução de custos, através da diminuição do retrabalho; redundâncias e erros nos processos; e a melhora da satisfação dos usuários, através da simplificação e automatização dos processos que acarreta em aumento da produtividade. Nessa dimensão, foram citados outros sistemas relacionados ao ERP que auxiliam nos benefícios transacionais, como, por exemplo, sistemas logísticos e da gestão da cadeia de suprimentos.

Os benefícios informacionais foram considerados importantes pelos respondentes, podendo-se destacar o acesso mais rápido e facilitado às informações, maior acuracidade da informação, possibilidade de prover as informações em diferentes formatos, o que, segundo os respondentes, auxilia no controle das atividades e melhora a tomada de decisão. Cabe ressaltar também, a questão da confiabilidade das informações e a utilização do ERP em conjunto com as práticas de BI. Na dimensão estratégica, os benefícios que o ERP proporciona estão relacionados principalmente ao alinhamento estratégico entre a $\mathrm{TI}$ e as diferentes áreas do negócio, sendo a $\mathrm{TI}$ fonte de criação de vantagem competitiva, melhores relações com clientes, que acarreta em aumento nas vendas e melhoria no posicionamento no mercado, através de segmentação dos clientes. Outro ponto importante destacado pelos gestores foi a utilização dos sistemas ERP de forma estratégica para auxiliar na inovação na empresa tanto nos processos quanto no mercado onde atua.

Além da identificação dos benefícios dos sistemas ERP, foi identificada a estruturação destes benefícios em cada uma das dimensões da GPTI. 
Pôde-se observar que os benefícios foram identificados em cada uma das dimensões nas três empresas de forma muito semelhante. As organizações 2 e 3 apresentaram os mesmos percentuais nas dimensões, enquanto na organização 1 foram observados mais benefícios informacionais. Também se observou que as três empresas percebem os benefícios estratégicos relacionados aos sistemas ERP de forma idêntica: 10\% dos benefícios analisados. No quadro 5 é apresentada a estruturação dos benefícios do ERP em cada uma das dimensões nas três organizações.

Percebe-se um benefício mais informacional do ERP na organização 1 . Esse aspecto foi identificado, pois a empresa utiliza mais esse sistema para o controle e gerenciamento tanto das informações e histórico dos pacientes, quanto das informações referentes a cirurgias e consultas realizadas pelos médicos. O benefício mais transacional encontrado nas organizações 2 e 3 diz respeito ao volume de produtos estocados e transacionados nestes tipos de organizações, e os dados necessários para a realização das atividades nessas empresas.

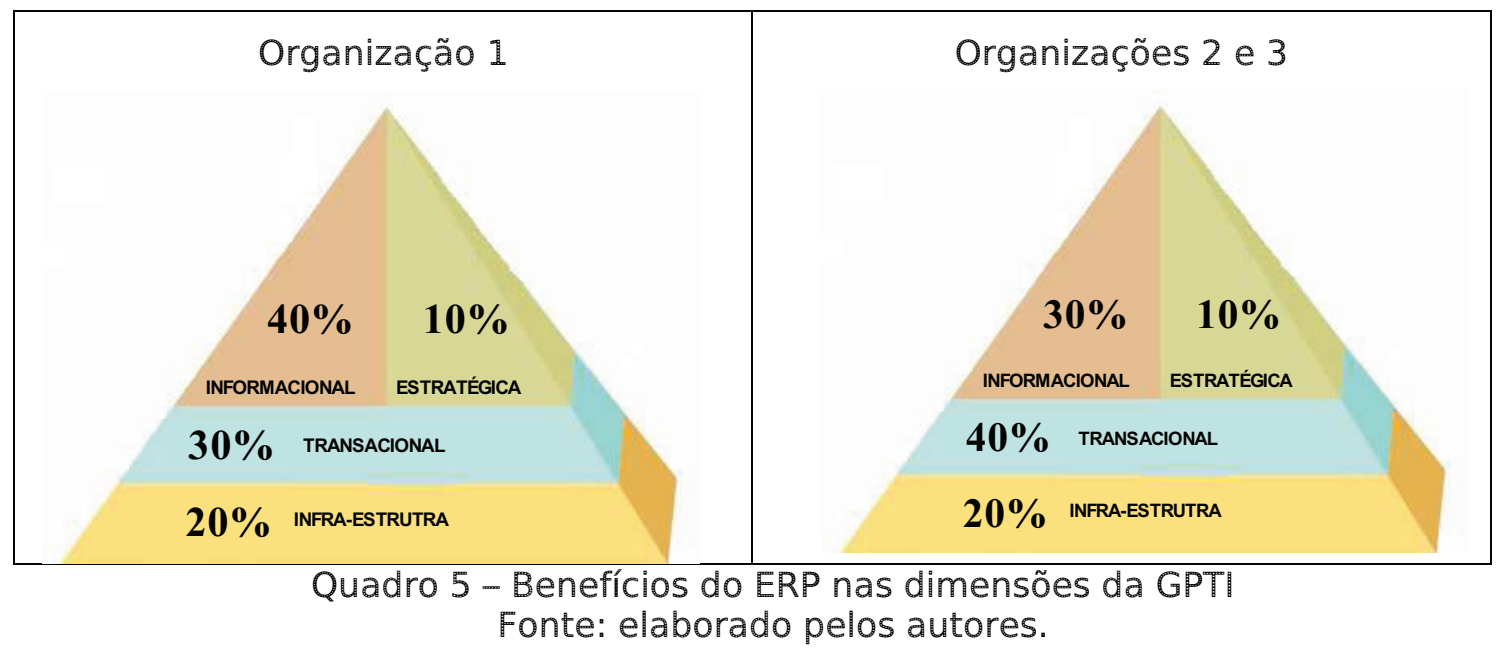

Em relação aos FCS, as três empresas apresentaram algumas diferenças, mas também algumas similaridades. A organização 1 acredita que os fatores críticos mais importantes estão relacionados à comunicação e cooperação, além do suporte da alta gerência e clareza dos objetivos/ metas. $\mathrm{Na}$ organização 2 , os fatores mais importantes também foram a cooperação e o suporte da alta gerência, incluindo a parceria com o fabricante, não mencionada na organização 1 . Na organização 3 , por sua vez, ficou mais evidente a necessidade de auxilio do fornecedor, através do uso de consultores, o que não tinha sido considerado tão importante nas outras empresas, além da presença da gestão das expectativas. Nas fases de implementação pôde-se destacar: (1) iniciação - escolha da arquitetura e clareza dos objetivos e metas foram os mais importantes; (2) adoção - suporte da alta gerência e parceria com o fabricante; (3) adaptação - as empresas percebem bem diferente essa fase, com fatores bem diferenciados, apenas pode-se citar a análise e conversão dos dados; (4) aceitação - suporte da alta gerência e a relação interdepartamental; (5) 
rotina - comunicação e relação com o fornecedor (suporte e parcerias); e (6) infusão - comunicação, cooperação e suporte da alta direção.

\section{CONSIDERAÇÕES FINAIS}

Nesta pesquisa foi identificado como as empresas podem analisar os investimentos nos sistemas ERP, examinando os benefícios que esses sistemas podem proporcionar, além de analisar os principais fatores críticos de sucesso. A combinação dos FCS com as dimensões da GPTI foi importante para atingir os resultados da pesquisa, pois evidencia uma nova forma para os gestores de $\mathrm{Tl}$ e de negócio melhor entender o processo de implementação de sistemas ERP.

Segundo a revista Computerworld (2009) um dos desafios das implementações de sistemas ERP é convencer as pessoas dos benefícios de ter um sistema de gestão unificado, que exige esforços de diversas unidades de negócio. Dessa forma, a utilização dos benefícios das dimensões da GPTI: infraestrutura, transacional, informacional e estratégica (WEILL e BROADBENT, 1998; WEILL e ARAL, 2006; DOLCl, 2009) pôde auxiliar as empresas nessa difícil tarefa, que demanda de meses a anos a ser completada, que é a implementação de sistemas ERP. As porcentagens dentro de cada uma das dimensões nas três empresas ficaram muito semelhantes.

Pode-se destacar que as empresas 2 e 3 consideram os sistemas ERP mais transacionais, correspondendo a $40 \%$ dos benefícios. Os benefícios operacionais dos sistemas ERP resultam da automatização dos processos entre as funções, do uso de dados para melhorar o plano e gestão da produção, e da monitoração e controle financeiro do desempenho de produtos, clientes, linhas de negócio e áreas de negócios, permitindo uma melhor relação com clientes, fornecedores, bancos, funcionários e transportadores (DOLCl, 2009a; SU e YANG, 2010). Enquanto a empresa 1, por sua vez, considera o sistema ERP mais informacional do que as demais dimensões, correspondendo a $40 \%$ dos benefícios. Essa percepção mais informacional corrobora a afirmação de Davenport (2002), de que os sistemas ERP são uma "fábrica de informações".

Os esforços das unidades de negócio podem ser auxiliado pela análise dos fatores críticos de sucesso (SOMERS e NELSON, 2001), merecendo destaque o suporte dos gestores, a comunicação e cooperação interdepartamental, além da necessidade de um líder do projeto que realize essa interação entre as diferentes áreas e a área de TI, que está realizando a implementação do sistema. Outra questão importante, abordada pelos FCS, é a relação entre a empresa fornecedora e a empresa que está adquirindo o sistema. Os gestores enfatizaram a necessidade do suporte de vendas, a questão das customizações, as ferramentas dos vendedores e o custo do uso dos consultores. Esses fatores foram considerados pelas três empresas no momento de identificar o fornecedor que mais se 
enquadrava nas necessidades das empresas analisadas que estivessem dentro dos limites financeiros de cada uma.

A partir dos resultados encontrados nesta pesquisa, sugere-se como pesquisa futura, realizar uma survey em diferentes empresas de diferentes setores para analisar como são percebidos os benefícios dos sistemas ERP em cada uma das dimensões da GPTI, bem como quais são os principais FCS dentro das diferentes fases da implementação dos sistemas.

\section{REFERÊNCIAS}

ARAL, S.; WEILL, P. IT assets, organizational capabilities and firm performance: asset and capability specific complementarities. Center for IS Research. Technical Research Report, 2004.

ARCHER, N. P.; GHASEMZADEH, F. An integrated framework for project portfolio selection. International journal of Project Management, v. 17, n. 4, p. 207-216, 1999.

BENBASAT, I.; GOLDSTEIN, D. K.; MEAD, M. The case research strategy in studies of information systems. MIS Quarterly, v. 11, n. 3, p. 369-386, 1987.

BERCHET, C.; HABCHI, G. The implementation and deployment of an ERP system: an industrial case study. Computers in Industry, v. 56, n. 6, p. 588-605, 2005.

BROADBENT, M,; WEILL, P. Management by maxim: how business and IT managers can create IT infrastructures. Sloan Management Review, v. 38, n. 3, p. 77-92, 1997.

BROADBENT, M.; WEILL, P.; NEO, B. S. Strategic context and patterns of infrastructure capability. Journal of Strategic Information Systems, v. 8, n. 2, p. 157-187, 1999.

CHEN, I. J. Planning for ERP systems: analysis and future trends. Business Process Management Journal, v. 7, n. 5, p. 374-386, 2001.

CHEN, S. G.; LIN, Y. K. On performance evaluation of ERP systems with fuzzy mathematics. Expert Systems with Applications, v. 36, n. 3, p. 63626367, 2009.

CHO, W.; SHAW, M. J. Balancing the strategic value and the operational value in IT portfolio selection, 2009. In: Americas Conference on Information Systems, São Francisco. Proceedings... Disponível em: http://aisel.aisnet.org/amcis2009/183. Acesso em: 20/10/2009.

CHO, W. IT Portfolio selection and IT synergy, 2009. In: Americas Conference on Information Systems, São Francisco. Proceedings.... Disponível em: http://aisel.aisnet.org/amcis2009_dc/1. Acesso em: 20/10/2009.

COMPUTERWORLD, 2009. Votorantim investe US\$ 53 milhões na padronização do ERP, 2009. Disponível em: http://cio.uol.com.br/gestao/ 
2009/10/30/votorantim-investe-us-53-milhoes-na-padronizacao-do-erp. Acesso em: 15/11/2009.

COOPER, R. B.; ZMUD, R. W. Information technology implementation research: a technological diffusion approach", Management Science, v. 36, n. 2, p. 123-139, 1990.

DAVENPORT, T. H. Missão crítica: obtendo vantagem competitiva com os sistemas de gestão empresarial. Porto Alegre: Bookman, 2002.

DAVENPORT, T. Putting the enterprise into the enterprise system. Harvard Business Review, v. 76, n. 4, p. 113-121, 1998.

DOLCI, P. C. Uso da gestão do portfólio de TI no processo de gerenciamento e justificativa dos investimentos em Tecnologia da Informação. 2009. Dissertação - Escola de Administração, Universidade Federal do Rio Grande do Sul, Porto Alegre, 2009a.

DOLCl, P. D. Explorando as dimensões da gestão do portfólio de TI (GPTI) com gestores de $\mathrm{TI}$ em empresas brasileiras. In: Encontro da ANPAD, XXXIII, São Paulo. Anais... ANPAD: 2009. CD-ROM.

DUBÉ, L.; PARÉ, G. Rigor in information systems positivist case research: current practices, trends and recommendations. MIS Quarterly, v. 27, n. 4, p. 597-635, 2003.

GLIEDMAN, C. The many faces of IT portfolio management. Research Digest, v. 5, n. 3, p. 1-10, 2002.

HOLSAPPLE, C. W.; SENA, M. P. ERP plans and decision-support benefits. Decision Support Systems, v. 38, n. 4, p. 575-590, 2005.

KARHADE, P. P.; SHAW, M.; SUBRAMANYAM, R. Evaluation of decision rules used for IT portfolio management: an inductive approach, 2009. . In: Americas Conference on Information Systems, São Francisco. Proceedings... Disponível em: http://aisel.aisnet.org/amcis2009/154. Acesso em: 22/10/2009.

$\mathrm{KOH}$, S. C. L.; GUNASEKARAN, A.; RAJKUMAR, D. ERPII: the involvement, benefits and impediments of collaborative information sharing. International Journal of Production Economics, v. 113, n. 1, p. 245-268, 2008.

LAUDON, K.; LAUNDON, J. Sistemas de informação gerenciais: administrando a empresa digital. 5. ed., São Paulo: Pearson, 2004.

LMBARROS, 2009. ERP nas 1.000 maiores empresas brasileiras - 2009. Disponível em: http://www.lbarros.com.br/artigos/ERP-2009.pdf. Acesso em: 10/12/2009.

MAIZLISH, B.; HANDLER, R. IT portfolio management step-by-step: unlocking the business value of IT. New Jersey: John Wiley \& Sons, Inc., 2005.

MALHOTRA, R.; TEMPONI, C. Critical decisions for ERP integration: small business issues. International journal of Information Management, v. 30, n. 1, p. 28-37, 2010.

MAZUTTI, C.; MAÇADA, A. C. G.; RIOS, L. R. O impacto do ERP na gestão da cadeia de suprimentos: estudo de caso em empresas do mercado 
brasileiro. In: Simpósio de Administração da Produção, Logística e Operações Internacionais, VIII, São Paulo. Anais... SIMPOI: 2005. CD-ROM. MCFARLAN, W. F. Portfolio approach to information systems. Harvard Business Review, v. 59, n. 5, p.142-150, 1981.

MEDEIROS JUNIOR, A. Seleção de sistema integrado de gestão (ERP) alinhado à estratégica de negócio. In: Encontro da ANPAD, XXXIII, São Paulo. Anais... ANPAD: 2009. CD-ROM.

MORTON, N. A.; HU, Q. Implications of the fit between organizational structure and ERP: a structural contingency theory perspective. International Journal of Information Management, v. 28, n. 5, p. 391-402, 2008.

RIEGE, A. M. Validity and reliability tests in case study research: a literature review with "hands-on" applications for each research phase. Qualitative Market Research, v. 6, n. 2, p. 78-86, 2003.

SCHNIEDERJANS, M. J.; HAMAKER, J. L.; SCHNIEDERJANS, A. M. Information Technology Investment: Decision-Making Methodology. Singapure: World Scientific Publishing Co., 2004.

SOMERS, T. M.; NELSON, K. The impact of critical success factors across the stages of enterprise resource planning implementations. In: Hawaii International Conference on System Sciences, $34^{\text {th }}$, Havaí. Proceedings... HICSS: 2001. CD-ROM.

SOUZA, C. A.; ZWICKER, R. Gestão de sistemas ERP: uma análise das capacidades e atores envolvidos. In: Encontro da ANPAD, XXX, São Paulo. Anais... ANPAD: 2006. CD-ROM.

SU, Y.; YANG, C. A structural equation model for analyzing the impact of ERP on SCM. Expert Systems with Applications, v. 37, n. 1, p. 456-469, 2010.

SUMNER, M. Critical success factors in enterprise wide information management systems. In: Hawaii International Conference on System Sciences, 32 ${ }^{\text {th }}$, Havaí. Proceedings... HICSS: 1999. CD-ROM.

SYMONS, C.; ORLOV, L. M.; BRIGHT, S.; BROWN, B. Optimizing the IT portfolio for maximum business value. Best Practices. Forrester Institute. p. 1-17, September, 2005.

TI INSIDE. Gartner reduz projeção mundial de gastos com ERP, 2008. Disponível em: http://www.tiinside.com.br/lmprimir.aspx? ID $=108437$. Acesso em: 01/05/2010.

TURBAN, E.; MCLEAN, E.; WETHERBE, J. C. Tecnologia da informação para gestão. 3. ed., Porto Alegre: Bookman, 2004.

TURNER, J.; LUCAS, H. C. Developing strategic information systems. In: W. Guth. Handbook of Business Strategy. Boston, Warren, Gorham e Lamont, 1985.

WANG, E. T. G.; SHIH, S.; JIANG, J. J.; KLEIN, G. The consistency among facilitating factors and ERP implementation success: a holistic view of fit. The fournal of Systems and Software, v. 81, n. 9, p. 1609-1621, 2008. 
WEILL, P.; ARAL, S. Generating premium returns on your IT investments. MIT Sloan Management Review, v. 47, n. 2, p. 38-48, 2006.

WEILL, P.; BROADBENT, M. Leveraging the new infrastructure: how market leaders capitalize on information technology. Boston: Harvard Business School Press, 1998.

WEILL, P.; SUBRAMANI, M.; BROADBENT, M. Building IT infrastructure for strategic agility. MIT Sloan Management Review, v. 44, n. 1, p. 56-65, 2002.

WEILL, P. The relationship between investment in information technology and firm performance: a study of the valve manufacturing sector. Information Systems Research, v. 3, n. 4, p. 307-333, 1992.

YIN, R. K. Estudo de caso - planejamento e métodos. 3. ed. Porto Alegre: Bookman, 2005.

ZHENG, G. A visual exploration approach to project portfolio management, 2007. In: Americas Conference on Information Systems, Keystone. Proceedings... Disponível em: http://aisel.aisnet.org/amcis2007/382. Acesso em: 20/10/2009. 